\title{
Absence of adverse hepatic or renal effects with the etonogestrel-releasing contraceptive implant inserted immediately postpartum
}

This article was published in the following Dove Press journal:

Open Access Journal of Contraception

14 November 2010

Number of times this article has been viewed

\author{
Milena B Brito ${ }^{1,2}$ \\ Rui A Ferriani, ${ }^{1,2}$ \\ Silvana M Quintana' \\ Marcos F Silva de Sál,2 \\ Carolina S Vieira ${ }^{1,2}$ \\ 'Department of Gynecology and \\ Obstetrics, University of Sao Paulo, \\ Ribeirao Preto School of Medicine, \\ Ribeirão Preto, Brazil; ${ }^{2}$ National \\ Institute of Hormones and Women's \\ Health, Ribeirão Preto, Brazil
}

Objective: To evaluate the safety, in terms of renal and hepatic function, of the etonogestrel (ENG)-releasing contraceptive implant inserted immediately postpartum.

Methods: A total of 40 healthy women were randomized; 20 received the ENG-releasing implant inserted 24 to 48 hours after delivery (ENG group), and 20 received depot medroxyprogesterone acetate in the sixth week postpartum (control group). Alkaline phosphatase, gamma-glutaryl transferase, alanine aminotransferase, aspartate aminotransferase, total bilirubin and its fractions, albumin, urea, and creatinine were analyzed.

Results: There was no statistically significant difference between the groups in serum hepatic markers or urea levels during the study. Creatinine levels increased in both groups during the first six weeks, more significantly in the ENG group than in the control group (ENG $+40 \%$ versus control $+20 \%, P=0.04$ ). This increase was followed by a greater decrease in the ENG group than in the control group 6-12 weeks postpartum (ENG $-14.3 \%$ versus control $-3.8 \%, P=0.02$ ). However, these changes were still within the normal ranges for the assays performed.

Conclusion: The ENG-releasing contraceptive implant inserted immediately postpartum was not associated with clinically significant changes in serum markers of liver or kidney function.

Keywords: etonogestrel, renal, hepatic, postpartum period, contraception, implant

\section{Introduction}

Progestin-only contraceptive methods are a widely recommended hormonal contraceptive option in the postpartum period for both nursing and nonnursing women. The World Health Organization recommends the use of progestin-only contraceptives by nursing women starting at six weeks after delivery, due to a theoretic risk to the child of exposure to steroid hormones during the first six weeks of life. ${ }^{1}$ Nonetheless, it has been recommended that the use of these contraceptives be initiated immediately postpartum in patients at a high risk for increased morbidity or mortality with a subsequent pregnancy and/or who experience difficulty accessing health care. ${ }^{1,2}$

The etonogestrel (ENG)-releasing contraceptive implant (NV Organon, Oss, The Netherlands) is a highly effective (Pearl Index $=0.38$ ), reversible, progestin-only contraceptive method. ${ }^{3}$ The long-acting characteristic of this method (three years) essentially eliminates adherence and user dependence from the effectiveness equation. ${ }^{4,5}$ The main side effect associated with this implant is a change in menstrual bleeding patterns, which is responsible for $14.4 \%$ of its removal during the first year of use. ${ }^{3}$ Other side effects associated with the implant include headache $(15.5 \%)$, weight gain $(12 \%)$, acne $(11.5 \%)$, breast tenderness $(10 \%)$, and emotional lability $(5.8 \%) .{ }^{3}$ ENG is
Correspondence: Carolina Sales Vieir Avenida Bandeirantes, 3900, Campus Universitário, Monte Alegre, Ribeirão Preto, SP, Brasil, CEP-|4049-900

Tel +55 I63602 282I

Fax +551636330946

Email carol.sales@uol.com.br 
the active metabolite of desogestrel, and its safety for the development of breastfeeding infants and its safety and effectiveness in lactating women have already been established. ${ }^{6-8}$ However, few data are available about the potential risks of inserting the implant immediately after childbirth.

Numerous physiologic adjustments occur after delivery as a woman's metabolism returns to its prepregnancy state. It is important that contraceptives offered during this time of intense physical change do not have deleterious effects on maternal health. We have previously demonstrated that the ENG-releasing implant did not significantly alter patients' lipid or carbohydrate metabolism, complete blood count, or inflammatory profile when inserted during the immediate postpartum period. ${ }^{8}$ The implant also produced no negative effects on newborn weight or on the maintenance of exclusive breastfeeding. ${ }^{8}$

Sex steroids affect hepatic function, but this effect is thought to be reduced when the drugs are not administered by the oral route. ${ }^{1}$ There have been no studies assessing renal and hepatic function when the ENG-releasing implant is administered during the immediate postpartum period, an interesting time to begin its use, especially for women at risk for short intergestational intervals. Thus, it is important to determine whether changes in renal and hepatic function occur when the ENG-releasing implant is inserted during the immediate postpartum period, as compared with the depot medroxyprogesterone acetate (DMPA) which is a contraception option more commonly prescribed in the post-puerperal period (starting at six weeks postpartum).

\section{Materials and methods}

We conducted a prospective, randomized, open clinical study comparing the maternal metabolic effects of two contraceptive regimens (see http://www.clinicaltrials.gov/, NCT00828542). In the first regimen, an ENG-releasing contraceptive implant was inserted during the immediate postpartum period; in the second, DMPA was initiated six weeks after delivery. Women using both regimens were studied for a 12-week period. The protocol was approved by the Institutional Review Board of the Ribeirão Preto School of Medicine, University of São Paulo, Brazil.

This report is a substudy of a protocol with the objective of evaluating maternal and neonatal safety after the early insertion of the ENG-releasing implant. Some results of this protocol have been previously reported. ${ }^{8}$ The inclusion criteria were age 18-35 years, desire to breastfeed, and interest in receiving long-acting contraception after childbirth. Exclusion criteria included being a smoker, being alcoholic or using recreational drugs, a body mass index $\left(\mathrm{kg} / \mathrm{m}^{2}\right) \geq 30$, systemic disease (diabetes mellitus, cardiovascular disease, liver disease, thyroid disease, or autoimmune disease), history (personal or family) of thromboembolic events, presenting alterations in hepatic enzymes, and being allergic to local anesthetic. All women in the prenatal care program were invited to participate in this study. These exclusion criteria resulted in the exclusion of three of 46 selected study participants due to hepatic enzyme alterations and the exclusion of one further patient due to obesity. Two women were lost to the study before the randomization process because they moved to another address. Thus, a total of 40 volunteers were enrolled in the study after giving written informed consent to participate. The sample size calculation has been previously presented. ${ }^{8}$

The women were assigned to two groups (in a 1:1 ratio) and randomized using a computer program (GraphPad Software, San Diego, CA). Twenty were assigned to use the ENG-releasing implant (ENG group) inserted 24 to 48 hours after delivery and 20 received no contraceptive method for six weeks after delivery (control group). At the end of this period, DMPA 150 mg (EMS Sigma Pharma, Hortolândia, Brazil) was administered intramuscularly to the control group. Women received follow-up visits at six and 12 weeks after delivery (see Figure 1).

Evaluation during the immediate postpartum period was performed in the morning at the hospital 24 to 48 hours after delivery and following a 12-hour fast. Peripheral blood samples $(20 \mathrm{~mL})$ were collected and processed within two hours. After serum was obtained from clotted blood, the samples were centrifuged at room temperature for 10 minutes, and the sera were stored at $-80^{\circ} \mathrm{C}$ until use. All variables were determined at the same time. Women were instructed to fast for 12 hours prior to follow-up visits and to arrive at the hospital in the morning, when blood was drawn and serum isolated by the procedure described above. The liver markers analyzed were gamma-glutamyl transferase $(\gamma-\mathrm{GT})$, alkaline phosphatase (AP), total bilirubin (TB), direct (DB) and indirect (IB) bilirubin, albumin, aspartate aminotransferase (AST), and alanine aminotransferase (ALT). The renal markers analyzed were creatinine and urea. All markers were determined using the Konelab 60i analyzer (Thermo Fisher Scientific, Espoo, Finland). The modified Szasz method was used for $\gamma$-GT determination using the Wiener ${ }^{\circledR}$ kinetic $\gamma$-GT test kit AA (Wiener Lab, Rosario, Argentina). AP was determined by the optimized kinetic method using the ALP 405 Wiener liquid AA kit (Wiener Lab). Albumin was determined by colorimetry using the Albumin AA kit 


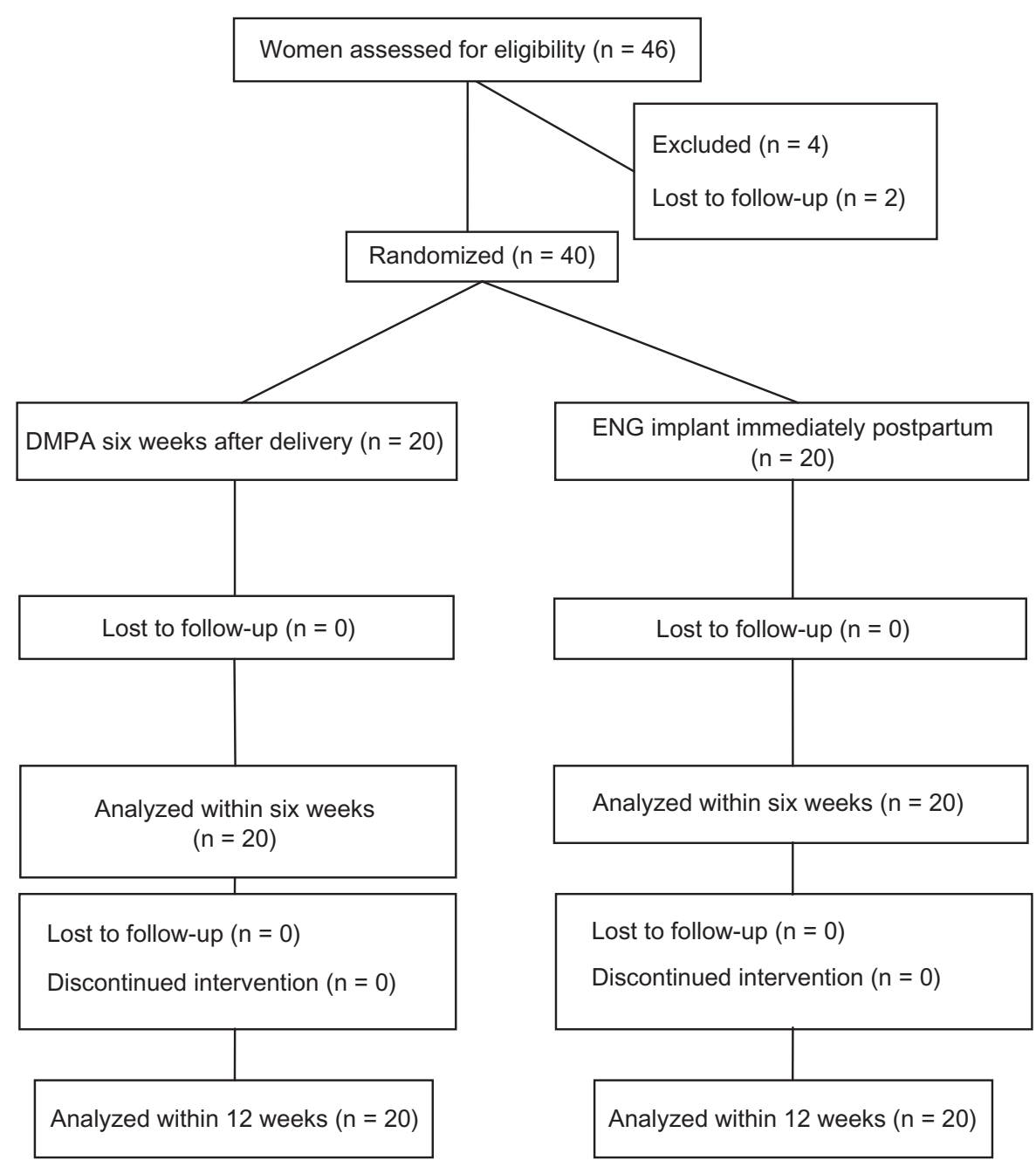

Figure I Flow chart of study participants.

Abbreviations: DMPA, depot medroxyprogesterone acetate; ENG, etonogestrel.

(Wiener Lab). TB and DB were measured by photometric testing using the Bilirubin Auto Total FS kit (DiaSys Diagnostic Systems GmbH, Holzheim, Germany) and the Direct Bilirubin FS kit (DiaSys Diagnostic Systems GmbH), respectively, and IB was calculated by subtracting the DB value from the TB value. The hepatic enzymes AST and ALT were determined by the optimized ultraviolet method (IFCC) using the AST liquid AA Wiener kit (Wiener Lab) and the ALT liquid AA Wiener kit (Wiener Lab). Creatinine and urea were determined by kinetic methods using the kinetic creatinine AA Wiener kit (Wiener Lab) and the kinetic UV urea AA Wiener kit (Wiener Lab), respectively.

\section{Statistical analysis}

The data were tested for normality using the KolmogorovSmirnov and Shapiro-Wilk tests. For intergroup comparisons, continuous variables with normal distributions were compared using unpaired Student's $t$-test; variables that were continuous but not normally distributed were compared using the Mann-Whitney $U$ test. The Fisher's exact test or the Chi-squared test was used to analyze categoric variables. The level of significance was set at $5 \%$. The data were analyzed statistically with SPSS 16.0 for Windows (SPSS Inc., Chicago, IL) and GraphPad 5.0 for Windows (GraphPad Software).

\section{Results}

The clinical and epidemiologic baseline characteristics of both groups were similar. There was no difference between the groups with regard to age, parity, route of delivery, family income, schooling, newborn weight, or prior breastfeeding (Table 1).

Although women were advised to avoid sexual intercourse until the first return visit, $40 \%$ of the women in the 
Table I Baseline epidemiologic characteristics of the study subjects

\begin{tabular}{|c|c|c|c|}
\hline & ENG group & Control group & $P$ \\
\hline Age (years, mean $\pm S D$ ) & $22.5 \pm 2.5$ & $22.9 \pm 4.4$ & $0.75^{\mathrm{a}}$ \\
\hline \multicolumn{4}{|l|}{ Current delivery } \\
\hline Vaginal & $80 \%(16 / 20)$ & $85 \%(17 / 20)$ & \multirow[t]{2}{*}{$1.00^{\mathrm{b}}$} \\
\hline Cesarean & $20 \%(4 / 20)$ & $15 \%(3 / 20)$ & \\
\hline \multicolumn{4}{|l|}{ Parity } \\
\hline I & $35 \%(7 / 20)$ & $60 \%(12 / 20)$ & \multirow[t]{3}{*}{$0.28^{c}$} \\
\hline 2 & $40 \%(8 / 20)$ & $25 \%(5 / 20)$ & \\
\hline$\geq 3$ & $25 \%(5 / 20)$ & $15 \%(3 / 20)$ & \\
\hline \multicolumn{4}{|l|}{ Family income } \\
\hline$<2$ minimum wages & $45 \%(9 / 20)$ & $40 \%(8 / 20)$ & \multirow[t]{2}{*}{$1.00^{\mathrm{d}}$} \\
\hline 2-6 minimum wages & $55 \%(11 / 20)$ & $60 \%(12 / 20)$ & \\
\hline \multicolumn{4}{|l|}{ Schooling } \\
\hline$<8$ years & $20 \%(4 / 20)$ & $25 \%(5 / 20)$ & \multirow[t]{3}{*}{$0.15^{\mathrm{e}}$} \\
\hline $8-11$ years & $70 \%(14 / 20)$ & $65 \%(13 / 20)$ & \\
\hline$\geq 12$ years & $10 \%(2 / 20)$ & $10 \%(2 / 20)$ & \\
\hline Previous breastfeeding & $60 \%(12 / 20)$ & $40 \%(8 / 20)$ & $0.34^{f}$ \\
\hline $\begin{array}{l}\text { Newborn's weight (g) } \\
\text { (mean } \pm \text { SD) }\end{array}$ & $3248 \pm 422$ & $3143 \pm 543$ & $0.50^{\mathrm{g}}$ \\
\hline
\end{tabular}

Notes: a,gUnpaired Student's t-test; b,d,fFisher's Exact test; ${ }^{c, e}$ Chi-squared test. Abbreviations: SD, standard deviation; ENG, etonogestrel.

ENG group and $45 \%$ in the control group reported having had sexual intercourse before the end of this six-week period.

During the immediate postpartum period, the variables did not differ between the two groups (Table 2). Changes in the serum markers are reported in two time intervals, ie, as the variation between baseline and six weeks after delivery (comparison of ENG-releasing implant with no hormonal contraception) and the variation between six and 12 weeks postpartum (comparison of ENG-releasing implant with DMPA).

\section{Variation between baseline and six weeks}

During the first six weeks after delivery there were elevations in serum $\gamma$-GT, ALT, DB, albumin, and urea in both groups and a decline in AP in both groups, with no difference between the groups. The levels of AST (ENG $+12.2 \%$ versus control $-13.7 \%, P=0.075)$ and $\mathrm{IB}(\mathrm{ENG}+27.3 \%$ versus control $-4.4 \%, P=0.1$ ) increased in the ENG group and decreased in the control group, although no statistical significant difference between the groups was observed. TB levels did not change in either group during this period (Table 3). The only marker that differed significantly between the groups during this period was creatinine, levels of which were increased in the ENG group compared with the control group $(\mathrm{ENG}+40 \%$ versus control $+20 \%, P=0.04)$.

\section{Variation between six and 12 weeks}

Reductions in AP and AST and increases in $\gamma$-GT and TB and its fractions were observed in both groups from six to 12 weeks postpartum. However, there was no difference between the groups (Table 3). During this period, the levels of albumin $(\mathrm{ENG}-2.7 \%$ versus control $+2.3 \%, P=0.10)$ and ALT (ENG $-5.5 \%$ versus control $+0.3 \%, P=0.69)$ decreased in the ENG group and increased in the control group. However, these differences were not significant (Table 3). As in the first six-week period, the only variable that differed significantly between the groups was creatinine, which was markedly reduced in the ENG group between weeks 6-12, in contrast with its trend in the $0-6$-week period (ENG $-14.3 \%$ versus control $-3.8 \% ; P=0.02$ ).

\section{Discussion}

The main finding of this study was that use of the ENGreleasing contraceptive implant immediately postpartum did not affect hepatic markers. With regard to renal variables, the implant caused a greater increase in creatinine levels than was seen in the control group during the first six weeks after delivery, and it also led to a greater reduction in creatinine

Table 2 Baseline values of hepatic and renal markers of healthy study subjects during immediate postpartum period

\begin{tabular}{|c|c|c|c|c|}
\hline Variables & Normal range & $\begin{array}{l}\text { ENG group } \\
\text { mean }( \pm S D)\end{array}$ & $\begin{array}{l}\text { Control group } \\
\text { mean }( \pm \text { SD) }\end{array}$ & $\begin{array}{l}\text { Intergroup } \\
\text { comparison }\left(P^{*}\right)\end{array}$ \\
\hline $\mathrm{AP}(\mathrm{U} / \mathrm{L})$ & $65-300$ & $400.3(137.6)$ & 335.5 (99.6) & 0.10 \\
\hline$\gamma$-GT (U/L) & $1 \mathrm{I}-50$ & $24.35(23.2)$ & $18.35(7.5)$ & 0.28 \\
\hline AST (U/L) & $<38$ & $23.8(7.4)$ & $26.6(8.4)$ & 0.27 \\
\hline ALT (U/L) & $<4$ I & $10.5(3.7)$ & $13.8(7.3)$ & 0.08 \\
\hline TB (mg/dL) & $0.2-1.2$ & $0.37(0.16)$ & $0.37(0.16)$ & 0.90 \\
\hline $\mathrm{DB}(\mathrm{mg} / \mathrm{dL})$ & $0-0.3$ & $0.14(0.07)$ & $0.14(0.06)$ & 1.00 \\
\hline IB (mg/dL) & $<1.2$ & $0.22(0.11)$ & $0.23(0.10)$ & 0.86 \\
\hline Albumin (g/dL) & $3.5-4.8$ & $3.26(0.49)$ & $3.18(0.46)$ & 0.60 \\
\hline Creatinine $(\mathrm{mg} / \mathrm{L})$ & $0.7-1.5$ & $0.65(0.11)$ & $0.65(0.16)$ & 1.00 \\
\hline Urea $(g / L)$ & $10-50$ & $17.6(5.62)$ & $19.85(7.46)$ & 0.28 \\
\hline
\end{tabular}

Notes: *P, Unpaired Student's t-test.

Abbreviations: ENG, etonogestrel-releasing implant; SD, standard deviation; AP, alkaline phosphatase; $\gamma$-GT, gamma-glutamyl transferase; AST, aspartate aminotransferase; ALT, alanine aminotransferase; TB, total bilirubin; DB, direct bilirubin; IB, indirect bilirubin. 
Table 3 Comparison of hepatic and renal markers between the group receiving etonogestrel in the immediate postpartum period and the control group during the first 12 weeks after delivery

\begin{tabular}{|c|c|c|c|c|}
\hline \multirow[t]{2}{*}{ Variables } & \multicolumn{2}{|l|}{$\Delta 0-6$ weeks } & \multicolumn{2}{|l|}{$\Delta 6-12$ weeks } \\
\hline & $\begin{array}{l}\text { Absolute variation } \\
\text { mean (SD) }\end{array}$ & $\%$ change from baseline & $\begin{array}{l}\text { Absolute variation } \\
\text { mean (SD) }\end{array}$ & $\%$ change from 6 weeks \\
\hline \multicolumn{5}{|l|}{ AP (U/L) } \\
\hline Implant & $-119.50(119.05)$ & $-29.8 \%$ & $-20.05(42.45)$ & $-7.2 \%$ \\
\hline DMPA & $-75.35(110.15)$ & $-22.5 \%$ & $-27.2(43.89)$ & $-10.4 \%$ \\
\hline Implant × DMPA $(P)$ & 0.23 & & 0.60 & \\
\hline \multicolumn{5}{|l|}{$\gamma$-GT $(\mathrm{U} / \mathrm{L})$} \\
\hline Implant & $+0.65(23.16)$ & $+2.7 \%$ & $+1.95(13.77)$ & $+7.8 \%$ \\
\hline DMPA & $+1.25(7.65)$ & $+12.1 \%$ & $+0.80(5.23)$ & $+4.1 \%$ \\
\hline Implant × DMPA $(P)$ & 0.91 & & 0.73 & \\
\hline \multicolumn{5}{|l|}{ AST (U/L) } \\
\hline Implant & $+2.90(12.14)$ & $+12.2 \%$ & $-3.30(9.33)$ & $-12.3 \%$ \\
\hline DMPA & $-3.65(10.42)$ & $-13.7 \%$ & $-2.40(7.74)$ & $-10.5 \%$ \\
\hline Implant × DMPA $(P)$ & 0.07 & & 0.74 & \\
\hline \multicolumn{5}{|l|}{$\operatorname{ALT}(\mathrm{U} / \mathrm{L})$} \\
\hline Implant & $+15.45(17.64)$ & $+52.9 \%$ & $-1.45(12.92)$ & $-5.5 \%$ \\
\hline DMPA & $+8.85(10.45)$ & $+64.1 \%$ & $+0.05(10.60)$ & $+0.3 \%$ \\
\hline Implant × DMPA $(P)$ & 0.16 & & 0.69 & \\
\hline \multicolumn{5}{|l|}{$\mathrm{TB}(\mathrm{mg} / \mathrm{dL})$} \\
\hline Implant & $+0.01(0.21)$ & $+2.7 \%$ & $+0.15(0.17)$ & $+32.6 \%$ \\
\hline DMPA & $-0.01(0.19)$ & $-2.7 \%$ & $+0.16(0.13)$ & $+43.2 \%$ \\
\hline Implant × DMPA $(P)$ & 0.12 & & 0.85 & \\
\hline \multicolumn{5}{|l|}{$\mathrm{DB}(\mathrm{mg} / \mathrm{dL})$} \\
\hline Implant & $+0.04(0.09)$ & $+28.5 \%$ & $+0.01(0.05)$ & $+5.6 \%$ \\
\hline DMPA & $+0.01(0.07)$ & $+7.2 \%$ & $+0.01(0.04)$ & $+6.7 \%$ \\
\hline Implant $\times$ DMPA $(P)$ & 0.23 & & 0.99 & \\
\hline \multicolumn{5}{|l|}{ IB (mg/dL) } \\
\hline Implant & $+0.06(0.14)$ & $+27.3 \%$ & $+0.14(0.14)$ & $+50 \%$ \\
\hline DMPA & $-0.01(0.13)$ & $-4.4 \%$ & $+0.15(0.1)$ & $+68 \%$ \\
\hline Implant $\times$ DMPA $(P)$ & 0.1 & & 0.79 & \\
\hline \multicolumn{5}{|l|}{ Albumin $(\mathrm{g} / \mathrm{dL})$} \\
\hline Implant & $+1.42(0.65)$ & $+43.5 \%$ & $-0.13(0.38)$ & $-2.7 \%$ \\
\hline DMPA & $+1.13(0.66)$ & $+35.5 \%$ & $+0.10(0.50)$ & $+2.35 \%$ \\
\hline Implant × DMPA $(P)$ & 0.17 & & 0.10 & \\
\hline \multicolumn{5}{|l|}{ Creatinine (mg/L) } \\
\hline Implant & $+0.26(0.18)$ & $+40 \%$ & $-0.13(0.15)$ & $-14.3 \%$ \\
\hline DMPA & $+0.13(0.19)$ & $+20 \%$ & $-0.03(0.12)$ & $-3.8 \%$ \\
\hline Implant × DMPA $(P)$ & $0.04 *$ & & $0.02 *$ & \\
\hline \multicolumn{5}{|l|}{ Urea $(g / L)$} \\
\hline Implant & + 12.85 (9.91) & $+72.8 \%$ & $+1.4(7.63)$ & $+4.8 \%$ \\
\hline DMPA & $+7.05(9.76)$ & $+35.7 \%$ & $+2.45(6.62)$ & $+9.1 \%$ \\
\hline Implant $\times$ DMPA $(P)$ & 0.07 & & 0.64 & \\
\hline
\end{tabular}

Notes: Data are expressed as mean variation ( \pm standard deviation) during the period evaluated. For statistical analyses, the Mann-Whitney $U$ test was used and $P<0.05$ was considered significant. $\Delta=$ variation between times. *Statistically significant.

Abbreviations: AP, alkaline phosphatase; $\gamma$-GT, gamma-glutamyl transferase; AST, aspartate aminotransferase; ALT, alanine aminotransferase; TB, total bilirubin; DB, direct bilirubin; IB, indirect bilirubin; ENG, etonogestrel; SD, standard deviation; DMPA, depot medroxyprogesterone acetate.

levels in the ENG group from weeks 6-12 postpartum. These changes were within the normal range for creatinine levels. Previous studies have not shown any adverse effects in renal or hepatic function in users of this implant inserted after the postpartum period. ${ }^{9-11}$ However, the present study was the first to assess the safety (measured by renal and hepatic markers) of early insertion of this implant before discharge from hospital after childbirth.
Contraception during the immediate postpartum period is important for family planning, especially in populations with limited access to health services or demonstrating low compliance with contraceptive methods. However, before prescribing a medication during the immediate postpartum period, it is necessary to determine its metabolic safety.

Renal function is affected by the physiologic increase in maternal blood volume during pregnancy, which is associated 
with reduced peripheral vascular resistance. There are increases in glomerular filtration and renal plasma flow during pregnancy. Additionally, there are changes in urea and creatinine levels, ${ }^{12}$ which return to pregravid levels 4-8 weeks after delivery. ${ }^{13}$ As expected, urea and creatinine levels initially increased in both groups as they returned to pregestational levels.

ENG is mainly excreted through urine. ${ }^{14} \mathrm{~A}$ small modification in renal function has been observed by Innal et al in a study of women who used the ENG-releasing implant for three years. ${ }^{10}$ The creatinine reduction observed in the previous study was attributed to a small increase in creatinine clearance or to tubular creatinine secretion independent of clearance; this is similar to the renal changes observed during the luteal phase of the menstrual cycle and attributed to progesterone. ${ }^{15}$ In the present study, insertion of the implant immediately after delivery was followed by a rise in creatinine levels, probably due to the reduction in blood volume during the postpartum period. However, at weeks 6-12 after delivery, there was a decline in creatinine levels, which was more pronounced in the ENG group. Similar findings have also been reported by other authors who evaluated the use of the ENG implant outside the immediate puerperium. ${ }^{10}$

Although there is no change in the general morphology of the liver during pregnancy, gestation is accompanied by changes in hepatic biochemical tests, such as reduction of serum albumin levels by hemodilution, discrete elevation of serum TB and hepatic enzymes close to delivery, elevation of $\gamma$-GT, and a marked elevation of AP due to the presence of placental AP. These values return to pregravid levels during the puerperium. ${ }^{16}$ Although the liver is not a target organ of sex steroids, it is influenced by them. The liver is inevitably more affected when steroids are administered by the oral route, due to the hepatic first-pass effect. The effects of the ENG-releasing implant on liver function have been previously studied outside the puerperium, and no clinically significant hepatic changes were observed during three years of medication use., ${ }^{9,11,17}$ Similarly, in the present investigation, although a greater increase in AST levels was observed in the ENG group during the first six weeks of the study, these levels remained within normal limits in all women, and no hepatic markers differed between the groups. A randomized study comparing 80 users of the ENG-releasing implant with users of the levonorgestrel (LNG)-releasing implant found elevations in TB, DB, and $\gamma$-GT levels compared with preinsertion levels among users of the ENG-releasing implant, although the values were within normal limits. AST levels increased during the first year of ENG implant use, then gradually returned to preinsertion levels. ${ }^{17}$
Another comparative study of ENG and LNG implants that assessed hepatic function for six months detected a significant elevation of TB and $\gamma$-GT and a reduction in AST and ALT. However, these changes were not clinically significant because the values remained within the normal range. ${ }^{9}$ The elevation of bilirubin levels detected in the present study agrees with previous reports, and is probably due to hepatic cholestasis induced by the hormone. ${ }^{9,11,17}$ AP values are quite high during the third trimester of pregnancy, and tend to fall to normal levels during the puerperium, as was the case for all the participants in both groups in the present study. A recent study assessing hepatic function and portal circulation in 50 users of the ENG-releasing implant for two years did not detect changes in portal blood circulation or in albumin levels, although there was an elevation in bilirubin levels and a decrease in hepatic enzymes with no clinical significance. ${ }^{11}$ Some authors have concluded that there may be mild hepatocellular dysfunction associated with the ENG-releasing implant, without clinical consequences for healthy users. ${ }^{17}$

Because it was a pilot study, the major limitation of the present trial was the possibility of underpowered analyses of the results. However, the changes in variables in ENG implant users were not clinically different from those observed in the control group. Another limitation is that only healthy volunteers were included in the study, so the findings in women with hepatic or renal disease could be different.

The immediate postpartum period represents a window of opportunity for contraception because a recent pregnancy and a new infant can provide strong motivation for the mother to use contraception. The urgency of contraceptive commencement is underscored by the resumption of sexual intercourse by almost half of the women in this study before the first medical visit after delivery. The hepatic and renal effects of the insertion of the ENG implant immediately after delivery did not lead to any deleterious alterations in this study. Given the findings of this study, the ENG-releasing contraceptive implant could be an interesting option for family planning, especially for women for whom delivery may represent the only opportunity to receive contraception.

\section{Acknowledgments}

The authors wish to thank all the participants in this study and Mrs Océlia Vasconcelos for invaluable collaboration with data collection. This study was supported by the Conselho Nacional de Pesquisa and the Fundação de Amparo à Pesquisa do Estado de São Paulo. 


\section{Disclosure}

The authors report no conflicts of interest in this work.

\section{References}

1. World Health Organization. Medical Eligibility Criteria for Contraceptive Use. 4th ed. Geneva, Switzerland: World Health Organization; 2009.

2. American Congress of Obstetricians and Gynecologists (ACOG) Committee Opinion No. 361: Breastfeeding: Maternal and infant aspects. Obstet Gynecol. 2007;109(2 Pt 1):479-480.

3. Darney P, Patel A, Rosen K, Shapiro LS, Kaunitz AM. Safety and efficacy of a single-rod etonogestrel implant (Implanon): Results from 11 international clinical trials. Fertil Steril. 2009;91(5):1646-1653.

4. Huber J. Pharmacokinetics of Implanon. An integrated analysis. Contraception. 1998;58 Suppl 6:85S-90S.

5. World Health Organization Department of Reproductive Health and Research (WHO/RHR) and Johns Hopkins Bloomberg School of Public Health/Center for Communication Programs (CCP), INFO Project. Family Planning: A Global Handbook for Providers (2008 update). Baltimore, MD: Center for Communication Programs and World Health Organization; 2008.

6. Reinprayoon D, Taneepanichskul S, Bunyavejchevin S, et al. Effects of the etonogestrel-releasing contraceptive implant (Implanon ${ }^{\circledR}$ ) on parameters of breastfeeding compared to those of an intrauterine device. Contraception. 2000;62(5):239-246.

7. Taneepanichskul S, Reinprayoon D, Thaithumyanon P, Praisuwanna P, Tosukhowong P, Dieben T. Effects of the etonogestrel-releasing implant Implanon and a nonmedicated intrauterine device on the growth of breast-fed infants. Contraception. 2006;73(4):368-371.
8. Brito MB, Ferriani RA, Quintana SM, Yazlle MED, Silva de Sá MF, Vieira CS. Safety of the etonogestrel-releasing implant during the immediate postpartum period: A pilot study. Contraception. 2009;80(6): 519-526.

9. Egberg N, van Beek A, Gunnervik C, et al. Effects on the hemostatic system and liver function in relation to Implanon and Norplant. A prospective randomized clinical trial. Contraception. 1998;58(2):93-98.

10. Inal MM, Yildirim Y, Ertopcu K, Avci ME, Ozelmas I, Tinar S. Effect of the subdermal contraceptive etonogestrel implant (Implanon) on biochemical and hormonal parameters (three years follow-up). Eur J Contracept Reprod Health Care. 2008;13(3):238-242.

11. Nasr A, Nafeh HM. Effect of etonogestrel contraceptive implant $\left(\right.$ Implanon $\left.^{\circledR}\right)$ on portal blood flow and liver functions. Contraception. 2009;9(3):236-239.

12. Lindheimer MD, Katz AI. Current concepts. The kidney in pregnancy. N Engl J Med. 1970;283(20):1095-1097.

13. Sims EAH, Krantz KE. Serial studies of renal function during pregnancy and the puerperium in normal women. J Clin Invest. 1958;37(12) 1764-1774.

14. Wenzl R, van Beek A, Schnabe P, Huber J. Pharmacokinetics of etonogestrel released from the contraceptive implant Implanon ${ }^{\circledR}$. Contraception. 1998;58(5):283-288.

15. Gault MH, Chafe L, Prabkaran V. Mid-menstrual cycle decline in creatinine and urea clearances. Nephron. 1994;67(2):1158-1166.

16. Duff P. Acute fatty liver of pregnancy. In: Clatek SL, editor. Critical Care Obstetrics. Boston, MA: Blackwell Scientific Publications; 1991.

17. Biswas A, Biswas S, Viegas OAC. Effect of etonogestrel subdermal contraceptive implant (Implanon ${ }^{\circledR}$ ) on liver function tests - a randomized comparative study with Norplant ${ }^{\mathbb{B}}$ implant. Contraception. 2004; 70:379-382.
Open Access Journal of Contraception

\section{Publish your work in this journal}

Open Access Journal of Contraception is an international, peerreviewed, open access, online journal, publishing original research, reports, reviews and commentaries on all areas of contraception. In addition to clinical research, demographics and health-related aspects, the journal welcomes new findings in animal and preclinical studies

\section{Dovepress}

relating to understanding the biological mechanisms and practical development of new contraceptive agents. The manuscript management system is completely online and includes a very quick and fair peer-review system. Visit http://www.dovepress.com/testimonials.php to read real quotes from published authors. 